${ }^{1}$ Facultad de Ciencias de la Salud, Universidad Autónoma de Chile, Talca, Chile.

2Departamento de Biología Celular, Facultad de Biología, Universidad de Barcelona, Barcelona, España. aLicenciado en Biología. PhD en Ciencias Biomédicas.

Recibido el 21 de enero de 2015, aceptado el 10 de junio de 2015.

Correspondencia a: Jordi Olloquequi. Facultad de Ciencias de la Salud Universidad Autónoma de Chile. 5 Poniente № 1670, 3460000 Talca, Chile.

Teléfono: +(56)(71)273-5728 jordiog82@gmail.com

\section{Efectores celulares de la respuesta inflamatoria en la enfermedad pulmonar obstructiva crónica}

\author{
RAFAEL SILVA O. ${ }^{1}$, JUAN F. MONTES ${ }^{2, a}$, \\ JOSÉ GARCÍA-VALERO ${ }^{2, a}$, JORDI OLLOQUEQUI ${ }^{1, a}$
}

\section{Cellular effectors of the inflammatory response in chronic obstructive pulmonary disease (COPD)}

Approximately 3 million people in the world die every year as a consequence of COPD, which is associated with an abnormal inflammatory response of the lung to noxious particles and gases. This inflammatory pattern causes pathological changes leading to a narrowing of small airways and destruction of lung parenchyma, also known as emphysema. Classically, these changes were associated to macrophages and neutrophils, although TCD8+ lymphocytes were latter added to the equation to explain the origin of emphysematous lesions. However, in recent years, multiple evidences have arisen indicating that inflammatory response in COPD is much more complex. These findings point to a key role for mast cells, dendritic cells, TCD4+ and B cells. The aim of this article is to review such evidence and report what is known so far about those cells involved in the inflammatory response in COPD.

(Rev Med Chile 2015; 143: 1162-1171)

Key words: Emphysema; Immunity; Inflammation; Leukocytes; Pulmonary disease, chronic obstructive.
L a enfermedad pulmonar obstructiva crónica (EPOC) es un proceso patológico de alta prevalencia, considerado en la actualidad la cuarta causa de muerte en el mundo y que, se estima, se convertirá en la tercera en el año $2030^{1}$. En los países de América Latina, 35 de cada 1.000 hospitalizaciones se deben a la EPOC, con un alto coste económico y con rangos de mortalidad intrahospitalaria que van de $6,7 \%$ a $29,5 \%{ }^{2}$. En Chile, las enfermedades respiratorias constituyen la tercera causa de muerte y, entre ellas, la EPOC ocupa el segundo lugar, representando a $22 \%$ de los decesos ${ }^{3}$. Por su parte, el Proyecto Latinoamericano de Investigación en Obstrucción Pulmonar (PLATINO) ha reportado que la prevalencia de la EPOC en Santiago de Chile es de 6,3\% en la población mayor de 40 años ${ }^{4}$.
La EPOC se define como una enfermedad caracterizada por una limitación al flujo aéreo no completamente reversible, progresiva y que se asocia a una respuesta inflamatoria anormal de los pulmones a partículas nocivas o gases ${ }^{5}$. Esta enfermedad comprende tanto los síntomas clínicos de la bronquitis/bronquiolitis crónica como los de la patología derivada del enfisema pulmonar. La confirmación diagnóstica se obtiene al realizar pruebas espirométricas, las cuales permiten determinar la obstrucción del flujo aéreo y el grado de alteración de la función pulmonar a partir del volumen espirado forzado en el primer segundo $\left(\mathrm{VEF}_{1}\right)$, la capacidad vital forzada (CVF) y la relación de estos dos parámetros clínicos $\left(\mathrm{VEF}_{1} / \mathrm{CVF}\right)^{5}$.

Se considera al humo del tabaco como el prin- 
cipal factor de riesgo para la EPOC 6 , aunque se han propuesto otros factores que pueden contribuir al desarrollo de esta enfermedad, entre los que destacan la contaminación del aire en ambientes interiores o exteriores, la exposición laboral a polvos y productos químicos, la recurrencia de infecciones respiratorias durante la niñez o la predisposición genética ${ }^{7}$.

A pesar de que se admite que la inflamación contribuye a la génesis de la EPOC, actualmente no existe una hipótesis patogénica clara que explique la manera en que esta inflamación influye en los cambios de la estructura pulmonar característicos de la enfermedad. El objetivo de este trabajo es revisar el rol de las principales células inflamatorias involucradas en la patogénesis de la EPOC.

\section{Efectores celulares inflamatorios en la EPOC}

La inflamación es una respuesta defensiva esencial que se activa ante diversas noxas y que tiene como objetivo mantener la homeostasis tisular frente a las condiciones nocivas generadas ${ }^{8}$. Esta respuesta tiene un papel clave, tanto en la defensa del organismo como en la génesis de desórdenes. Así, aunque la inflamación es un mecanismo necesario para eliminar infecciones, reparar daños tisulares y suprimir la iniciación o progresión de tumores, la inflamación crónica puede contribuir a la patogénesis de enfermedades muy diversas 9 .

La inflamación de las vías aéreas constituye un rasgo común para la EPOC y otras enfermedades obstructivas de gran prevalencia, como el asma o la bronquiectasia. Sin embargo, el patrón inflamatorio de estas enfermedades presenta diferentes características $^{10,11}$. En el asma, los eosinófilos, los mastocitos y los linfocitos $\mathrm{T} \mathrm{CD}^{+}{ }^{+}$representan las células inflamatorias protagonistas del proceso inflamatorio. Por el contrario, en la EPOC y la bronquiectasia, se han reportado densidades mayores de neutrófilos, macrófagos y linfocitos $\mathrm{T}$ $\mathrm{CD}^{+}$. En la EPOC, además, el proceso inflamatorio pulmonar va más allá de las vías aéreas y afecta al parénquima, lo que contribuye a la formación de enfisema.

Por otro lado, el proceso inflamatorio en la EPOC evoluciona a medida que la enfermedad progresa. La inflamación empieza mucho antes de que los pacientes experimenten los síntomas, e incluso los fumadores asintomáticos sufren una inflamación pulmonar significativa cuando se les compara con los no-fumadores ${ }^{12}$. Además, a pesar de que dejar de fumar tiene un efecto beneficioso para los pacientes con EPOC, la inflamación persiste mucho después de haber abandonado el hábito tabáquico ${ }^{13,14}$. Es por ello que se considera que el proceso inflamatorio en la EPOC es una amplificación en cuanto a la intensidad y duración de aquél que es característico en fumadores sin la enfermedad.

Así pues, la EPOC se caracteriza por presentar un patrón inflamatorio específico y complejo, que incluye tanto respuestas de la inflamación aguda como de la crónica, y que se traduce en una gran diversidad de los efectores celulares y de los mediadores pro-inflamatorios que regulan este proceso (Figura 1).

\section{Neutrófilos}

Los neutrófilos son células de la primera línea defensiva del sistema inmunitario y sus funciones más importantes incluyen la fagocitosis de microorganismos patógenos y la regulación del proceso inflamatorio agudo. También constituyen una fuente de especies reactivas del oxígeno, de citoquinas pro-inflamatorias (como la interleuquina [IL]-8), de mediadores lipídicos (como el leucotrieno-B4), de péptidos antibacterianos (como las defensinas) y de enzimas que pueden dañar los tejidos (como la elastasa humana de neutrófilos o la catepsina-G $)^{15-17}$. Los neutrófilos han sido asociados con la aparición de metaplasia de células mucosas en la bronquitis crónica, así como con el aumento de la contractibilidad de la musculatura lisa en las vías y también con la destrucción de tejido pulmonar en el enfisema ${ }^{18}$. En relación a esto último, estudios recientes han descrito correlaciones entre los niveles en sangre de la fracción soluble de ICAM-1, una proteína de adhesión esencial para el reclutamiento de los neutrófilos a los órganos, y un empeoramiento en la función pulmonar ${ }^{19} \mathrm{y}$ la progresión del enfisema ${ }^{20}$.

Algunos estudios han analizado la densidad de estas células en bronquiolos y en parénquima pulmonar de enfermos de EPOC, aunque los resultados no han sido concluyentes. Así, algunos autores han descrito un incremento de la densidad neutrofílica en bronquiolos ${ }^{21,22}$ y parénquima pulmonar $^{22,23}$ de enfermos de EPOC comparados con individuos sin la enfermedad. Nuestro grupo de investigación reportó, además, una correlación 


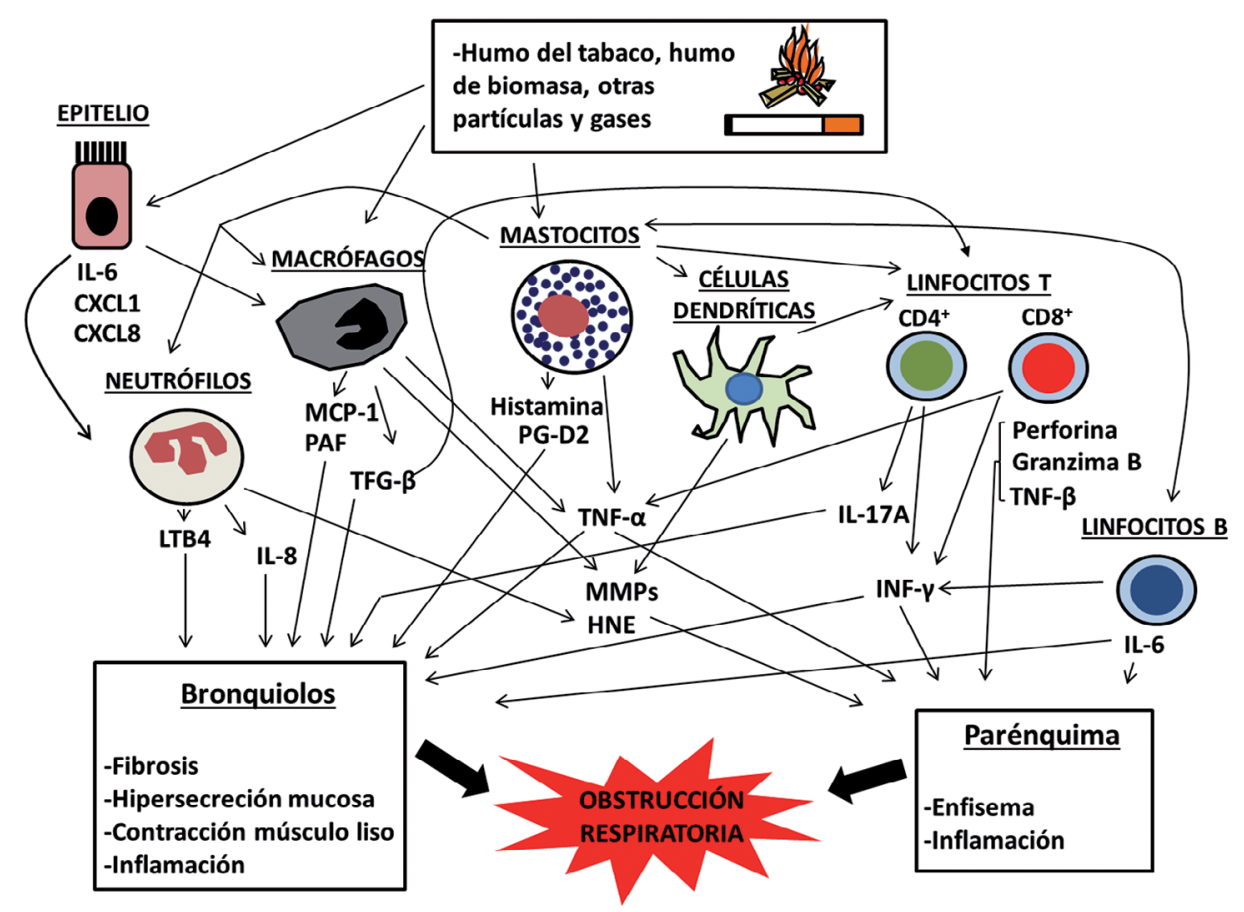

Figura 1. Células inflamatorias y sus principales mediadores químicos en la EPOC. La respuesta inflamatoria característica de la EPOC engloba células de la inmunidad innata y de la adquirida, las cuales, a través de sus mediadores químicos, se regulan entre sí y perpetúan un estado inflamatorio que influye en los cambios histopatológicos que se observan en la enfermedad. En las etapas iniciales de la respuesta, agentes como el humo de tabaco, el humo de biomasa u otros gases y partículas activarían a células de la primera línea defensiva como el epitelio respiratorio, los macrófagos, los mastocitos y los neutrófilos. En aquellos individuos susceptibles de padecer EPOC, la respuesta inflamatoria se perpetuaría e incluiría la participación de células mediadoras de la inmunidad adquirida como los linfocitos. Así, mediante la secreción de mediadores como el PAF, por parte de macrófagos, y la histamina o la PG-D2, por parte de mastocitos, se incrementa la contracción de la musculatura lisa en bronquiolos, estrechando su lumen. La HNE, el TGF- $\beta$ o la IL-17A, producidos por neutrófilos, macrófagos y linfocitos $\mathrm{TCD}^{+}$, respectivamente, contribuyen a la hipersecreción mucosa en vías aéreas. Por su parte, mediadores como el TNF- $\alpha$, la IL-6, la IL-17A, el MCP-1, la IL-8, el LTB4 o el INF- $\gamma$ favorecen la infiltración y/o la activación de diversas células inflamatorias en bronquiolos y parénquima pulmonar. Moléculas como la HNE, secretada por neutrófilos, y las MMPs, producidas por macrófagos y células dendríticas, pueden dañar directamente la matriz extracelular y contribuir a la formación de enfisema. La acción citolítica de la perforina, la granzima B o el TNF- $\beta$, producidos por los linfocitos TCD8 ${ }^{+}$, también puede tener un papel en la patogenia del enfisema causando la apoptosis de células epiteliales y fibroblastos de paredes alveolares. Finalmente, las moléculas derivadas de la ruptura de la matriz extracelular o aquellas modificadas por la acción del humo del tabaco podrían convertirse en autoantígenos que, a través del concurso de células dendríticas, linfocitos B y linfocitos T CD4 ${ }^{+}$propiciarían un desorden autoinmune, tal y como ha sido propuesto por algunos autores. IL-6: interleuquina 6 ; CXCL 1: ligando quimioquina CXC 1; CXCL 8: ligando quimioquina CXC 8; LTB4: leucotrieno B4; IL-8: interleuquina 8; MCP-1: proteína quimioatrayente de monocitos-1; PAF: factor activador de plaquetas; TFG- $\beta$ : factor de crecimiento transformante; PG-D2: prostaglandina D2; TNF- $\alpha$ : factor de necrosis tumoral $\alpha$; MMPs: metaloproteasas de matriz; HNE: elastasa de neutrófilos humana; IL-17A: interleuquina 17A; INF- $\gamma$ : interferón $\gamma$; TNF- $\beta$ : factor de necrosis tumoral $\beta$ o linfotoxina.

entre la densidad de neutrófilos en dichos compartimentos pulmonares y un descenso del $\mathrm{VEF}_{1}$, así como un incremento del grado de enfisema macroscópico ${ }^{22}$. Estos resultados se contraponen a los de otros estudios que no han encontrado diferencias significativas en la cantidad de neutrófilos infiltrados en bronquiolos ${ }^{24-26}$ y parénquima ${ }^{27}$ pulmonar entre enfermos y no enfermos de EPOC. Dichas discordancias podrían ser un reflejo de la corta duración de la vida de los neutrófilos infiltrados en un tejido y de su rápido tránsito a través de las vías aéreas y el parénquima pulmonar ${ }^{18}$, lo que provoca una gran variabilidad en las estimaciones de las densidades de estas células. 


\section{Macrófagos}

Los macrófagos son células con un papel clave tanto en la inflamación aguda como en la crónica y representan la mayoría de células inflamatorias presentes en el tracto respiratorio de individuos fumadores y no fumadores ${ }^{28,29}$. Son células efectoras de larga vida y dotadas de gran capacidad reactiva, ya que responden a estímulos endógenos y exógenos, pero también son proactivas, dado que producen mediadores que modulan la acción de las células que los rodean. De este modo, los macrófagos se convierten en un elemento esencial en todas las respuestas inflamatorias, ya sea fagocitando tanto organismos patógenos, como eliminando partículas inhaladas y restos de células necróticas o apoptóticas.

Los macrófagos pueden secretar más de 100 sustancias diferentes, implicadas en la activación del movimiento, crecimiento y diferenciación celulares, así como en la regulación del crecimiento de vasos sanguíneos y la modificación de las estructuras formadas por tejido conjuntivo ${ }^{30}$. Entre estas sustancias destacan factores quimiotácticos (como la MCP-1), citoquinas pro-inflamatorias (como la IL-8 o el factor de necrosis tumoral [TN$\mathrm{F}]-\alpha$ ), constrictores de la musculatura lisa (como el factor activador de plaquetas [PAF]), activadores de la secreción de las glándulas mucosas (como el factor transformador de crecimiento [TGF]- $\beta$ ) y proteínas de la matriz extracelular (como la fibronectina o la decorina $)^{31-33}$. Además, son también una fuente de metaloproteasas de matriz (MMPs) que, al combinarse entre sí, pueden degradar un espectro de moléculas similar al de las proteasas de los neutrófilos ${ }^{34} \mathrm{y}$ también facilitar la migración y la infiltración de los leucocitos hacia tejidos dañados ${ }^{35}$. De entre todas las MMPs, la MMP-2, la MMP-9 y la MMP-12 (también conocida como elastasa de los macrófagos) son las que más se han relacionado con la patogénesis de la $\mathrm{EPOC}^{36}$.

Se ha demostrado una mayor densidad de estas células en las vías aéreas y en el parénquima de los enfermos de EPOC, comparados con individuos sin la enfermedad ${ }^{14,23,26,37}$. Además, se ha descrito una correlación entre la cantidad de macrófagos infiltrados en el subepitelio bronquial y un descenso del $\mathrm{VEF}_{1}{ }^{38}$, mientras que la densidad de los macrófagos infiltrados en las paredes alveolares ha mostrado una correlación positiva con la gravedad del enfisema ${ }^{39}$. Estos datos son relevantes, ya que un estudio reciente en un modelo experimental de EPOC ha demostrado que una disminución del número de macrófagos en los pulmones confiere protección ante el desarrollo de enfisema ${ }^{40}$.

Por otro lado, diversos trabajos ponen de manifiesto que los macrófagos en la EPOC no sólo se incrementan en número, sino que su función difiere en comparación a los de aquellos sujetos con una función pulmonar normal ${ }^{41}$. Así, se ha reportado que los macrófagos de los enfermos de EPOC presentan una menor capacidad para fagocitar microorganismos y células apoptóticas ${ }^{42,43}$, además de alteraciones en la diferenciación hacia un fenotipo pro o anti-inflamatorio ${ }^{44}$.

\section{Mastocitos}

Los mastocitos actúan como células centinelas del sistema inmunitario y lo hacen con una gran eficiencia, dado que tienen receptores capaces de detectar casi cualquier factor de lesión ${ }^{45}$. Se encuentran ampliamente distribuidos por todos los tejidos vascularizados, donde normalmente residen cerca de vasos sanguíneos, de nervios, células de la musculatura lisa, de glándulas mucosas y de folículos pilosos, y también son especialmente abundantes en aquellos lugares que están en contacto directo con el medio externo, como la piel, las vías aéreas y el tracto gastrointestinal ${ }^{46}$.

Aunque los mastocitos son bien conocidos por su función efectora en procesos alérgicos, durante los últimos años se ha caracterizado un amplio abanico de funciones inmunitarias que estas células pueden desarrollar y que van desde la iniciación y el mantenimiento de la inflamación aguda, hasta la activación de las respuestas de la inmunidad adquirida ${ }^{45}$. Esto es así gracias a su capacidad para producir y secretar un amplio abanico de mediadores activos (como la histamina, los proteoglicanos y las proteasas neutras), de citoquinas y quimioquinas (como el TNF- $\alpha$ o la limfotactina), y de mediadores lipídicos (como la prostaglandina [PG]-D2), entre otros ${ }^{47,48}$, cuyo papel inmunoregulador sobre diferentes tipos celulares es muy importante. Además, se ha demostrado que el humo del tabaco puede inducir la secreción de algunos de estos mediadores químicos por parte de los mastocitos in vitro, lo cual hace factible que la infiltración de efectores inflamatorios que se observa en pulmones de fumadores esté mediada por estas células ${ }^{49}$.

En los pulmones de individuos sanos, los mastocitos están presentes en todos los compar- 
timentos histológicos ${ }^{50}$. A pesar de que la gran capacidad inflamatoria de estas células las convierte en elementos potencialmente relevantes en la patogénesis de la EPOC, sólo hay un trabajo que demuestre una densidad significativamente superior de mastocitos en bronquiolos de enfermos de EPOC $^{51}$, mientras que varios autores han mostrado que no existen diferencias significativas en el número de mastocitos infiltrando bronquios ${ }^{14,52,53}$, bronquiolos $^{22}$ o parénquima ${ }^{27,54}$ de enfermos y no enfermos de EPOC. Además, algunos autores han reportado una reducción significativa de la densidad total de mastocitos tanto en bronquios ${ }^{55}$ como en bronquiolos y vasos pulmonares ${ }^{50} \mathrm{de}$ enfermos de EPOC comparados con individuos no enfermos, e incluso se ha encontrado una correlación positiva entre el número de mastocitos infiltrando bronquiolos y el $\mathrm{VEF}_{1}{ }^{55}$.

\section{Células dendríticas}

Las células dendríticas constituyen una población especializada de células mononucleares responsables del reconocimiento, la captación y la presentación de antígenos a los linfocitos, iniciando así una respuesta inmunitaria adaptativa ante patógenos ${ }^{56}$.

La implicación de estas células en la patogénesis de la EPOC plantea aún muchos interrogantes. Una hipótesis es que el humo del tabaco podría alterar la maduración y la función de las células dendríticas, afectando la capacidad de inducir una respuesta inmunitaria efectiva en el tracto respiratorio $^{57}$. Esta respuesta anormal de las células dendríticas favorecería la aparición del patrón de inflamación alterado característico de la EPOC y podría ser responsable, por ejemplo, de un incremento de la proliferación de los linfocitos $\mathrm{T} \mathrm{CD}^{+}$ o de fenómenos de autoinmunidad ${ }^{57}$.

En cuanto a las densidades de células dendríticas detectadas en pulmones de enfermos de EPOC, los resultados son contradictorios. Así, algunos autores han reportado un incremento en el número de células dendríticas maduras en tejido pulmonar de enfermos de $\mathrm{EPOC}^{58}$, mientras que otros describen una reducción significativa de estas células en bronquiolos y parénquima de sujetos con la enfermedad ${ }^{59}$. Otros estudios muestran que los fumadores con EPOC tienen 100 veces menos células dendríticas en el epitelio bronquial comparados con controles sanos y que los niveles de estas células se normalizan tras abandonar el hábito tabáquico ${ }^{60}$. Estos resultados son consistentes con aquellos descritos en modelos animales que muestran una disminución en el número y la activación de células dendríticas pulmonares tras la exposición a humo de tabaco ${ }^{61}$.

\section{Linfocitos $T C D 8^{+}$}

Los linfocitos $\mathrm{T} \mathrm{CD}^{+}$son una de las principales defensas del organismo frente a virus, bacterias intracelulares y procesos cancerosos, gracias a su acción citolítica sobre las células infectadas o malignas.

A pesar de ser el tipo de linfocito $\mathrm{T}$ predominante, tanto en las vías aéreas como en el compartimento alveolar de los enfermos de EPOC $^{62}$, el papel de los linfocitos $T$ citotóxicos en la enfermedad aún se desconoce. No obstante, su capacidad de dañar el tejido pulmonar las convierte en uno de los efectores celulares más relevantes en la patogénesis de la enfermedad. Así, los linfocitos T CD8 ${ }^{+}$ tienen la capacidad de causar citólisis y apoptosis de las células epiteliales alveolares por medio de la liberación de perforina, de granzima-B y de TNF- $\alpha^{63}$. De hecho, se ha descrito una asociación entre los linfocitos $\mathrm{T} \mathrm{CD} 8^{+}$y la apoptosis de células alveolares en el enfisema ${ }^{64} \mathrm{y}$ un estudio reciente, llevado a cabo en ratones, ha demostrado que se puede reducir la formación de enfisema mediante la inhibición de las células $\mathrm{CD} 8^{+65}$. Los linfocitos T citotóxicos también pueden producir una variedad de citoquinas del tipo 1 , entre las que se incluyen el TNF- $\alpha$, la linfotoxina (o TNF- $\beta$ ) y el interferón (INF)- $\gamma^{66}$. Además, algunos de los mediadores pro-inflamatorios de los linfocitos $\mathrm{T} \mathrm{CD}^{+}$pueden favorecer el proceso de fibrosis y engrosamiento de la capa de musculatura lisa que se observa en vías aéreas de individuos afectados de EPOC $^{67,68}$, incrementando la resistencia al flujo de aire.

En cuanto a la densidad de estas células en tejido pulmonar de enfermos de EPOC, en algunos estudios se ha observado un incremento en el número de linfocitos $\mathrm{T} \mathrm{CD}^{+}$tanto en bronquios $^{26,69}$ como en bronquiolos ${ }^{25,38,52,67,70} \mathrm{y}$ pared alveolar ${ }^{23,38,64,70}$ de individuos enfermos comparados con individuos sin la EPOC. Algunos autores, además, han observado una correlación significativa entre el aumento de linfocitos $\mathrm{CD}^{+}$ y la disminución del VEF1 $1^{38,67,69}$. Resultados de nuestro grupo de investigación pusieron de manifiesto que la densidad de linfocitos $\mathrm{T} \mathrm{CD}^{+}$en parénquima peri-enfisematoso de pacientes con 
EPOC era superior a la encontrada en parénquima no afectado, reforzando la idea de que estas células podrían tener un papel destacado en la génesis del enfisema ${ }^{70}$.

\section{Linfocitos T CD4 ${ }^{+}$}

Los linfocitos $\mathrm{T} \mathrm{CD}^{+}$son esenciales en la regulación de la actividad de otras células inmunitarias. Gracias a su capacidad de secretar un amplio abanico de citoquinas (como el IFN- $\gamma$ o la IL-9, entre muchas otras) los linfocitos $\mathrm{T} \mathrm{CD} 4^{+}$modulan el reclutamiento, la activación o la proliferación de células inmunocompetentes como los linfocitos $\mathrm{B}$, los linfocitos T CD8 ${ }^{+}$o los macrófagos ${ }^{71}$. Los pacientes con EPOC estable presentan una acumulación de, como mínimo, dos clases de linfocitos $\mathrm{T} \mathrm{CD}^{+}$en sus pulmones: los Th1 y los Th1 $17^{72,73}$.

Se ha reportado que los enfermos de EPOC presentan un mayor porcentaje de células Th1 y que secretan más IFN- $\gamma$ que en fumadores control $^{73}$. Además, en los pacientes con EPOC grave se ha observado una alta expresión de IL-18, citoquina que promueve el desarrollo de las células Th1 ${ }^{74}$. En cuanto a las células Th17, las cuales están implicadas en procesos autoinmunes y en el reclutamiento de otras células inflamatorias a tejidos $^{75}$, se encuentran incrementadas en sangre de enfermos de EPOC en comparación a fumadores control ${ }^{76}$. Por otro lado, también se ha reportado un incremento de células que expresan IL-17A (una de las principales interleuquinas producidas por las células Th17) en submucosa de pacientes con $\mathrm{EPOC}^{72}$.

En cuanto a la densidad de linfocitos T $\mathrm{CD}^{+}{ }^{+}$en pared alveolar, los datos disponibles son escasos. Retamales et al..$^{23}$ detectaron un aumento de estas células en paredes alveolares de fumadores con enfisema grave, al compararlos con individuos fumadores que presentaban enfisema leve o falta de enfisema. Aoshiba et al. ${ }^{77}$ observaron que el número de linfocitos $\mathrm{T} \mathrm{CD}^{+}{ }^{+}$era más elevado en las zonas con lesiones enfisematosas más graves, mientras que los $\mathrm{CD}^{+}$predominaban en zonas con un grado de enfisema moderado.

\section{Linfocitos B}

Los linfocitos B se caracterizan por su capacidad de producir inmunoglobulinas, moléculas capaces de unirse a antígenos específicos, pero también por secretar una amplia variedad de citoquinas, como IL-6, IL-10 o IFN- $\gamma^{78}$.
Aunque no se conoce el papel que podrían tener los linfocitos B en la patogénesis de la EPOC, se ha especulado que una acumulación de estas células podría ser el resultado de una reacción contra algún antígeno específico, el cual podría provenir de patógenos del aparato respiratorio ${ }^{67} \mathrm{o}$ incluso de algún componente del humo del tabaco ${ }^{79}$. En relación a esta idea, se ha desarrollado lo que se conoce como "la hipótesis autoinmune", la cual postula que uno de los mecanismos implicados en la patogénesis de la EPOC es una reacción inmunitaria contra alguno o algunos constituyentes del tejido pulmonar (por ejemplo, moléculas de la matriz extracelular) que se han visto modificados como consecuencia de la exposición prolongada al humo del tabaco ${ }^{80}$. Estas estructuras alteradas, podrían actuar como autoantígenos y perpetuar un estado inflamatorio crónico.

En cuanto a la presencia de estas células en pulmones de enfermos de EPOC, algunos estudios sostienen, por un lado, que la infiltración de linfocitos B es más importante en vías de individuos afectados por la enfermedad, y por otro, que esta infiltración se hace más importante a medida que la EPOC progresa ${ }^{24,67,79}$. En relación al parénquima pulmonar, los pocos estudios que se han realizado coinciden en afirmar que no existen diferencias significativas en la carga de linfocitos $\mathrm{B}$ infiltrados en paredes alveolares de individuos con y sin EPOC $^{23,39,70}$. No obstante, la presencia de agregados de linfocitos B formando folículos linfoides se ha observado tanto en vías aéreas como en parénquima de pacientes de EPOC $^{67,81,82}$. Respecto a estas estructuras, resultados de un trabajo reciente desarrollado en ratones sugieren que los linfocitos B presentes en folículos linfoides pulmonares son necesarios para inducir la activación de macrófagos alveolares y promover la aparición de enfisema ${ }^{83}$.

\section{Conclusiones}

La EPOC es una enfermedad compleja que cursa con una respuesta inflamatoria anormal, considerada una amplificación de la que es característica en aquellos fumadores que no desarrollan la enfermedad. Durante muchos años se ha pensado que la reacción inflamatoria que se da en los pulmones de los individuos fumadores estaba orquestada por neutrófilos y macrófagos, 
y que las enzimas elastasas de estas células eran las responsables de la destrucción pulmonar en la EPOC. En los últimos años, este concepto se ha ido revisando para englobar un proceso inflamatorio más amplio y que otorga un papel importante a otras células de la inmunidad innata y adquirida. Así, varios autores que han analizado los perfiles celulares en alvéolos y bronquiolos han demostrado un incremento de varios tipos de efectores celulares inflamatorios, incluyendo no sólo los macrófagos y los neutrófilos, sino también los mastocitos, las células dendríticas y los linfocitos.

Actualmente, los tratamientos que se aplican a los enfermos de EPOC se limitan a mitigar los síntomas y están basados fundamentalmente en el uso de broncodilatadores y de corticoides inhalados para reducir la respuesta inflamatoria. Sin embargo, encontrar nuevos fármacos capaces de frenar la progresión de la enfermedad o incluso de prevenir el declive de la función pulmonar, sólo será posible si somos capaces de bloquear determinados aspectos de los procesos inflamatorios y de remodelación tisular característicos de la EPOC. Para ello, es necesario entender cuál es el papel específico de cada uno de los tipos celulares involucrados en la patogénesis de la EPOC, reto que puede abrir la puerta a futuras dianas terapéuticas para combatir esta enfermedad.

Agradecimientos: Los autores agradecen a la Srta. Carmen Gloria Muñoz Pincheira su apoyo administrativo para la gestación de este artículo de revisión.

\section{Referencias}

1. World Health Organization (WHO). Chronic Respiratoy Diseases. Burden of COPD. Disponible en: http:// www.who.int/respiratory/copd/burden/en/ [Consultado el 12 de enero de 2015].

2. Ciapponi A, Alison L, Agustina M, Demian G, Silvana C, Edgardo S. The epidemiology and burden of COPD in Latin America and the Caribbean: systematic review and meta-analysis. COPD 2014; 11 (3): 339-50.

3. Szot MJ. Mortalidad por enfermedades respiratorias en Chile durante 1999. Rev Chil Enferm Respir 2003; 19: 8-14.

4. Baptista AM, Gomes C. Platino Study-Chilean Survey Report. Disponible en: http://www.platino-alat.org/ docs/report_platino_chile_2004.pdf [Consultado el 20 de diciembre de 2014].
5. Global Initiative for Chronic Lung Diseases (GOLD). Global Strategy for the Diagnosis, Management and Prevention of COPD. Disponible en: http://www.goldcopd. org/ [Consultado el 11 de noviembre de 2014].

6. Vestbo J, Hurd SS, Agusti AG, Jones PW, Vogelmeier C, Anzueto A, et al. Global strategy for the diagnosis, management, and prevention of chronic obstructive pulmonary disease: GOLD executive summary. Am J Respir Crit Care Med 2013; 187 (4): 347-65.

7. Eisner MD, Anthonisen N, Coultas D, Kuenzli N, Perez-Padilla R, Postma D, et al. An official American Thoracic Society public policy statement: Novel risk factors and the global burden of chronic obstructive pulmonary disease. Am J Respir Crit Care Med 2010; 182 (5): 693-718.

8. Medzhitov R. Origin and physiological roles of inflammation. Nature 2008; 454 (7203): 428-35.

9. Okin D, Medzhitov R. Evolution of inflammatory diseases. Curr Biol 2012; 22 (17): R733-40.

10. Athanazio R. Airway disease: similarities and differences between asthma, COPD and bronchiectasis. Clinics (Sao Paulo) 2012; 67 (11): 1335-43.

11. Sutherland ER, Martin RJ. Airway inflammation in chronic obstructive pulmonary disease: comparisons with asthma. J Allergy Clin Immunol 2003; 112 (5): 819-27; quiz 28.

12. Amin K, Ekberg-Jansson A, Lofdahl CG, Venge P. Relationship between inflammatory cells and structural changes in the lungs of asymptomatic and never smokers: a biopsy study. Thorax 2003; 58 (2): 135-42.

13. Wright JL, Lawson LM, Pare PD, Wiggs BJ, Kennedy S, Hogg JC. Morphology of peripheral airways in current smokers and ex-smokers. Am Rev Respir Dis 1983; 127 (4): 474-7.

14. Rutgers SR, Postma DS, Ten Hacken NH, Kauffman HF, van Der Mark TW, Koeter GH, et al. Ongoing airway inflammation in patients with COPD who Do not currently smoke. Chest 2000; 117 (5 Suppl 1): 262s.

15. Dubravec DB, Spriggs DR, Mannick JA, Rodrick ML. Circulating human peripheral blood granulocytes synthesize and secrete tumor necrosis factor alpha. Proc Natl Acad Sci USA 1990; 87 (17): 6758-61.

16. Tetley TD. New perspectives on basic mechanisms in lung disease. 6. Proteinase imbalance: its role in lung disease. Thorax 1993; 48 (5): 560-5.

17. Tecchio C, Micheletti A, Cassatella MA. Neutrophil-derived cytokines: facts beyond expression. Front Immunol 2014; 5: 508.

18. O’Donnell R, Breen D, Wilson S, Djukanovic R. Inflammatory cells in the airways in COPD. Thorax 2006; 61 (5): 448-54. 
19. Thyagarajan B, Smith LJ, Barr RG, Gross MD, Sood A, Kalhan R, et al. Association of circulating adhesion molecules with lung function. The CARDIA study. Chest 2009; 135 (6): 1481-7.

20. Aaron CP, Schwartz JE, Bielinski SJ, Hoffman EA, Austin JH, Oelsner EC, et al. Intercellular adhesion molecule 1 and progression of percent emphysema: The MESA Lung Study. Respir Med 2015; 109 (2): 255-64.

21. Pilette C, Godding V, Kiss R, Delos M, Verbeken E, Decaestecker C, et al. Reduced epithelial expression of secretory component in small airways correlates with airflow obstruction in chronic obstructive pulmonary disease. Am J Respir Crit Care Med 2001; 163 (1): 18594.

22. Olloquequi J, García-Valero J, Rodríguez E, Montero MA, Ferrer J, Montes JF. Lung CD57+ cell density is increased in very severe COPD. Histol Histopathol 2012; 27 (1): 39-47.

23. Retamales I, Elliott WM, Meshi B, Coxson HO, Pare PD, Sciurba FC, et al. Amplification of inflammation in emphysema and its association with latent adenoviral infection. Am J Respir Crit Care Med 2001; 164 (3): 469-73.

24. Bosken CH, Hards J, Gatter K, Hogg JC. Characterization of the inflammatory reaction in the peripheral airways of cigarette smokers using immunocytochemistry. Am Rev Respir Dis 1992; 145 (4 Pt 1): 911-7.

25. Lams BE, Sousa AR, Rees PJ, Lee TH. Immunopathology of the small-airway submucosa in smokers with and without chronic obstructive pulmonary disease. Am J Respir Crit Care Med 1998; 158 (5 Pt 1): 1518-23.

26. Turato G, Zuin R, Miniati M, Baraldo S, Rea F, Beghe B, et al. Airway inflammation in severe chronic obstructive pulmonary disease: relationship with lung function and radiologic emphysema. Am J Respir Crit Care Med 2002; 166 (1): 105-10.

27. Saetta M, Baraldo S, Corbino L, Turato G, Braccioni F, Rea F, et al. CD8+ve cells in the lungs of smokers with chronic obstructive pulmonary disease. Am J Respir Crit Care Med 1999; 160 (2): 711-7.

28. Linden M, Rasmussen JB, Piitulainen E, Tunek A, Larson $\mathrm{M}$, Tegner $\mathrm{H}$, et al. Airway inflammation in smokers with nonobstructive and obstructive chronic bronchitis. Am Rev Respir Dis 1993; 148 (5): 1226-32.

29. Kuschner WG, D’Alessandro A, Wong H, Blanc PD. Dose-dependent cigarette smoking-related inflammatory responses in healthy adults. Eur Respir J 1996; 9 (10): 1989-94.

30. Pappas K, Papaioannou AI, Kostikas K, Tzanakis N. The role of macrophages in obstructive airways disease: chronic obstructive pulmonary disease and asthma. Cytokine 2013; 64 (3): 613-25.

31. Nathan CF. Secretory products of macrophages. J Clin Invest 1987; 79 (2): 319-26.

32. Barnes PJ. Alveolar macrophages as orchestrators of COPD. COPD 2004; 1 (1): 59-70.

33. Murugan V, Peck MJ. Signal transduction pathways linking the activation of alveolar macrophages with the recruitment of neutrophils to lungs in chronic obstructive pulmonary disease. Exp Lung Res 2009; 35 (6): 439-85.

34. Davey A, McAuley DF, O'Kane CM. Matrix metalloproteinases in acute lung injury: mediators of injury and drivers of repair. Eur Respir J 2011; 38 (4): 959-70.

35. Kumagai K, Ohno I, Okada S, Ohkawara Y, Suzuki K, Shinya $\mathrm{T}$, et al. Inhibition of matrix metalloproteinases prevents allergen-induced airway inflammation in a murine model of asthma. J Immunol 1999; 162 (7): 4212-9.

36. Vlahos R, Bozinovski S. Role of alveolar macrophages in chronic obstructive pulmonary disease. Front Immunol 2014; 5: 435.

37. Saetta M, Turato G, Baraldo S, Zanin A, Braccioni F, Mapp CE, et al. Goblet cell hyperplasia and epithelial inflammation in peripheral airways of smokers with both symptoms of chronic bronchitis and chronic airflow limitation. Am J Respir Crit Care Med 2000; 161 (3 Pt 1): 1016-21.

38. Di Stefano A, Capelli A, Lusuardi M, Balbo P, Vecchio C, Maestrelli $\mathrm{P}$, et al. Severity of airflow limitation is associated with severity of airway inflammation in smokers. Am J Respir Crit Care Med 1998; 158 (4): 1277-85.

39. Finkelstein R, Fraser RS, Ghezzo H, Cosio MG. Alveolar inflammation and its relation to emphysema in smokers. Am J Respir Crit Care Med 1995; 152 (5 Pt 1): 1666-72.

40. Beckett EL, Stevens RL, Jarnicki AG, Kim RY, Hanish I, Hansbro NG, et al. A new short-term mouse model of chronic obstructive pulmonary disease identifies a role for mast cell tryptase in pathogenesis. J Allergy Clin Immunol 2013; 131 (3): 752-62.

41. Hiemstra PS. Altered macrophage function in chronic obstructive pulmonary disease. Ann Am Thorac Soc 2013; 10 Suppl: S180-5.

42. Berenson CS, Garlipp MA, Grove LJ, Maloney J, Sethi S. Impaired phagocytosis of nontypeable Haemophilus influenzae by human alveolar macrophages in chronic obstructive pulmonary disease. J Infect Dis 2006; 194 (10): 1375-84.

43. Hodge S, Hodge G, Ahern J, Jersmann H, Holmes M, Reynolds PN. Smoking alters alveolar macrophage recognition and phagocytic ability: implications in chronic 
obstructive pulmonary disease. Am J Respir Cell Mol Biol 2007; 37 (6): 748-55.

44. Shaykhiev R, Krause A, Salit J, Strulovici-Barel Y, Harvey BG, O'Connor TP, et al. Smoking-dependent reprogramming of alveolar macrophage polarization: implication for pathogenesis of chronic obstructive pulmonary disease. J Immunol 2009; 183 (4): 2867-83.

45. Metz M, Maurer M. Mast cells-key effector cells in immune responses. Trends Immunol 2007; 28 (5): 234-41.

46. Dawicki W, Marshall JS. New and emerging roles for mast cells in host defence. Curr Opin Immunol 2007; 19 (1): 31-8.

47. Galli SJ, Kalesnikoff J, Grimbaldeston MA, Piliponsky AM, Williams CM, Tsai M. Mast cells as "tunable" effector and immunoregulatory cells: recent advances. Annu Rev Immunol 2005; 23: 749-86.

48. Galli SJ, Nakae S, Tsai M. Mast cells in the development of adaptive immune responses. Nat Immunol 2005; 6 (2): 135-42.

49. Mortaz E, Folkerts G, Redegeld F. Mast cells and COPD. Pulm Pharmacol Ther 2011; 24 (4): 367-72.

50. Andersson CK, Mori M, Bjermer L, Lofdahl CG, Erjefalt JS. Novel site-specific mast cell subpopulations in the human lung. Thorax 2009; 64 (4): 297-305.

51. Grashoff WF, Sont JK, Sterk PJ, Hiemstra PS, de Boer WI, Stolk J, et al. Chronic obstructive pulmonary disease: role of bronchiolar mast cells and macrophages. Am J Pathol 1997; 151 (6): 1785-90.

52. Baraldo S, Turato G, Badin C, Bazzan E, Beghe B, Zuin $\mathrm{R}$, et al. Neutrophilic infiltration within the airway smooth muscle in patients with COPD. Thorax 2004; 59 (4): 308-12.

53. Willemse BW, Ten Hacken NH, Rutgers B, LesmanLeegte IG, Postma DS, Timens W. Effect of 1-year smoking cessation on airway inflammation in COPD and asymptomatic smokers. Eur Respir J 2005; 26 (5): 835-45.

54. Andersson CK, Mori M, Bjermer L, Lofdahl CG, Erjefalt JS. Alterations in lung mast cell populations in patients with chronic obstructive pulmonary disease. Am J Respir Crit Care Med 2010; 181 (3): 206-17.

55. Gosman MM, Postma DS, Vonk JM, Rutgers B, Lodewijk M, Smith M, et al. Association of mast cells with lung function in chronic obstructive pulmonary disease. Respir Res 2008; 9: 64.

56. Pearce EJ, Everts B. Dendritic cell metabolism. Nat Rev Immunol 2015; 15 (1): 18-29.

57. Tsoumakidou M, Demedts IK, Brusselle GG, Jeffery PK. Dendritic cells in chronic obstructive pulmonary disease: new players in an old game. Am J Respir Crit Care Med 2008; 177 (11): 1180-6.
58. Vassallo R, Walters PR, Lamont J, Kottom TJ, Yi ES, Limper AH. Cigarette smoke promotes dendritic cell accumulation in COPD; a Lung Tissue Research Consortium study. Respir Res 2010; 11: 45.

59. Tsoumakidou M, Koutsopoulos AV, Tzanakis N, Dambaki K, Tzortzaki E, Zakynthinos S, et al. Decreased small airway and alveolar CD83+ dendritic cells in COPD. Chest 2009; 136 (3): 726-33.

60. Rogers AV, Adelroth E, Hattotuwa K, Dewar A, Jeffery PK. Bronchial mucosal dendritic cells in smokers and ex-smokers with COPD: an electron microscopic study. Thorax 2008; 63 (2): 108-14.

61. Robbins CS, Pouladi MA, Fattouh R, Dawe DE, Vujicic $\mathrm{N}$, Richards CD, et al. Mainstream cigarette smoke exposure attenuates airway immune inflammatory responses to surrogate and common environmental allergens in mice, despite evidence of increased systemic sensitization. J Immunol 2005; 175 (5): 2834-42.

62. Brusselle GG, Joos GF, Bracke KR. New insights into the immunology of chronic obstructive pulmonary disease. Lancet 2011; 378 (9795): 1015-26.

63. Plataki M, Tzortzaki E, Rytila P, Demosthenes M, Koutsopoulos A, Siafakas NM. Apoptotic mechanisms in the pathogenesis of COPD. Int J Chron Obstruct Pulmon Dis 2006; 1 (2): 161-71.

64. Majo J, Ghezzo H, Cosio MG. Lymphocyte population and apoptosis in the lungs of smokers and their relation to emphysema. Eur Respir J 2001; 17 (5): 946-53.

65. Podolin PL, Foley JP, Carpenter DC, Bolognese BJ, Logan GA, Long E 3rd, et al. T cell depletion protects against alveolar destruction due to chronic cigarette smoke exposure in mice. Am J Physiol Lung Cell Mol Physiol 2013; 304 (5): L312-23.

66. Barnes PJ. Cellular and molecular mechanisms of chronic obstructive pulmonary disease. Clin Chest Med 2014; 35 (1): 71-86.

67. Hogg JC, Chu F, Utokaparch S, Woods R, Elliott WM, Buzatu L, et al. The nature of small-airway obstruction in chronic obstructive pulmonary disease. N Engl J Med 2004; 350 (26): 2645-53.

68. Baraldo S, Turato G, Saetta M. Pathophysiology of the small airways in chronic obstructive pulmonary disease. Respiration 2012; 84 (2): 89-97.

69. Lams BE, Sousa AR, Rees PJ, Lee TH. Subepithelial immunopathology of the large airways in smokers with and without chronic obstructive pulmonary disease. Eur Respir J 2000; 15 (3): 512-6.

70. Olloquequi J, Ferrer J, Montes JF, Rodríguez E, Montero MA, Garcia-Valero J. Differential lymphocyte infiltration in small airways and lung parenchyma in COPD patients. Respir Med 2010; 104 (9): 1310-8. 
71. Luckheeram RV, Zhou R, Verma AD, Xia B. CD4(+)T cells: differentiation and functions. Clin Dev Immunol 2012; 2012: 925135.

72. Di Stefano A, Caramori G, Gnemmi I, Contoli M, Vicari C, Capelli A, et al. T helper type 17-related cytokine expression is increased in the bronchial mucosa of stable chronic obstructive pulmonary disease patients. Clin Exp Immunol 2009; 157 (2): 316-24.

73. Grumelli S, Corry DB, Song LZ, Song L, Green L, Huh J, et al. An immune basis for lung parenchymal destruction in chronic obstructive pulmonary disease and emphysema. PLoS Med 2004; 1 (1): e8.

74. Imaoka $\mathrm{H}$, Hoshino $\mathrm{T}$, Takei $\mathrm{S}$, Kinoshita $\mathrm{T}$, Okamoto $\mathrm{M}$, Kawayama $\mathrm{T}$, et al. Interleukin-18 production and pulmonary function in COPD. Eur Respir J 2008; 31 (2): 287-97.

75. Kheradmand F, Shan M, Xu C, Corry DB. Autoimmunity in chronic obstructive pulmonary disease: clinical and experimental evidence. Expert Rev Clin Immunol 2012; 8 (3): 285-92.

76. Vargas-Rojas MI, Ramírez-Venegas A, Limon-Camacho L, Ochoa L, Hernández-Zenteno R, Sansores RH. Increase of Th17 cells in peripheral blood of patients with chronic obstructive pulmonary disease. Respir Med 2011; 105 (11): 1648-54.
77. Aoshiba K, Koinuma M, Yokohori N, Nagai A. Differences in the distribution of CD4+ and CD8+ T cells in emphysematous lungs. Respiration 2004; 71 (2): 184-90.

78. Mauri C, Bosma A. Immune regulatory function of B cells. Annu Rev Immunol 2012; 30: 221-41.

79. Gosman MM, Willemse BW, Jansen DF, Lapperre TS, van Schadewijk A, Hiemstra PS, et al. Increased number of B-cells in bronchial biopsies in COPD. Eur Respir J 2006; 27 (1): 60-4.

80. Agusti A, MacNee W, Donaldson K, Cosio M. Hypothesis: does COPD have an autoimmune component? Thorax 2003; 58 (10): 832-4.

81. Olloquequi J, Montes JF, Prats A, Rodríguez E, Montero MA, García-Valero J, et al. Significant increase of CD57+ cells in pulmonary lymphoid follicles of COPD patients. Eur Respir J 2011; 37 (2): 289-98.

82. Van der Strate BW, Postma DS, Brandsma CA, Melgert BN, Luinge MA, Geerlings M, et al. Cigarette smoke-induced emphysema: A role for the B cell? Am J Respir Crit Care Med 2006; 173 (7): 751-8.

83. John-Schuster G, Hager K, Conlon TM, Irmler M, Beckers J, Eickelberg O, et al. Cigarette smoke-induced iBALT mediates macrophage activation in a B cell-dependent manner in COPD. Am J Physiol Lung Cell Mol Physiol 2014; 307 (9): L692-706. 